\title{
Intuitive Mapping of Perfusion Parameters to Glyph Shape
}

\author{
Steffen Oeltze ${ }^{1}$, Arvid Malyszczyk ${ }^{1}$, Bernhard Preim ${ }^{1}$ \\ ${ }^{1}$ Institut für Simulation und Graphik, Otto-von-Guericke Universität Magdeburg \\ stoeltze@isg.cs.uni-magdeburg.de
}

\begin{abstract}
Perfusion data characterize the regional blood flow in human tissue. For each voxel, a time-intensity curve (TIC) represents the enhancement of a contrast agent (CA). Parameters describing the TIC shape, e.g. the area below the curve (1), maximum (2), and slope (3), characterize the perfusion and have to be derived and integrated for diagnosis. We present the integrated visualization of perfusion parameters based on their intuitive mapping to glyph shape. Glyph attributes such as size, vertical extension and orientation are modified according to (1), (2), and (3) respectively, thereby generating an easy to interpret coding of TIC shape. We further introduce a multi-resolution glyph display and couple our visualizations with a feature specification component.
\end{abstract}

\section{Introduction}

In perfusion imaging, the distribution of a contrast agent $(\mathrm{CA})$ is registered to assess blood flow and tissue kinetics. For each voxel, a time-intensity curve (TIC) characterizes the CA enhancement (Fig. 1). How long it takes until the maximum amount of CA is delivered (TTP), which maximum (Peak) is achieved, the velocity of CA wash-in (Slope), the overall amount of CA (Integral), the first momentum of the curve (MTT), as well as other parameters are derived voxelwise from these curves and stored in parameter volumes. The derived parameters are substitutes for physiological parameters such as tumor perfusion and vessel permeability [1]. The integrated analysis of several parameters is essential. For ischemic stroke diagnosis, e.g., if the blood flow is delayed in a particular region, it is crucial to evaluate if the overall blood flow is also reduced [2].

A straightforward method to analyze several parameters is the side-by-side presentation of color-coded parameter maps (slice in a parameter volume) [2]. However, this requires a mental integration of suspicious regions. Multiparameter visualizations, integrating several perfusion parameters in one image, were introduced in [3] and [4]. Apart from color icons though, none of the presented techniques facilitates the examination of the regional distribution of more than three parameters. However, in perfusion diagnosis, 5-7 relevant parameters may be derived from a TIC (Fig. 1). To accomplish an integrated analysis of the regional distribution of perfusion parameters, we adopt glyphs as a standard technique from the visualization of multi-field data. A glyph is a simple geometric primitive which is positioned with respect to the original data points in space 
and whose attributes, e.g., color, extension, size, and orientation, are modified according to the represented parameter values. Superquadric tensor glyphs have been applied for tensor field visualization based on data from diffusion tensor imaging [5]. In [6], color-coded ellipsoids are employed for visualizing the myocardial strain based on a model and finite element simulations. The glyph-based integrated visualization of multi-modal cardiac MR-data has been presented in [7]. In [8], a surface-based glyph placement strategy is proposed for the visualization of multi-modal volumetric datasets. Inspired by previous work, we present the integrated glyph-based visualization of perfusion parameters. We focus on an intuitive mapping of parameters to glyph attributes.

\section{Materials and Methods}

We applied our techniques to cerebral MR perfusion datasets and to Dynamic Contrast Enhanced (DCE) MR mammography datasets. The latter have been motion-corrected according to [9]. Parameter volumes have been pre-computed for all datasets and all relevant parameters (Fig. 1). The presented techniques have been integrated in the MeVisLab platform (www.mevislab.de).

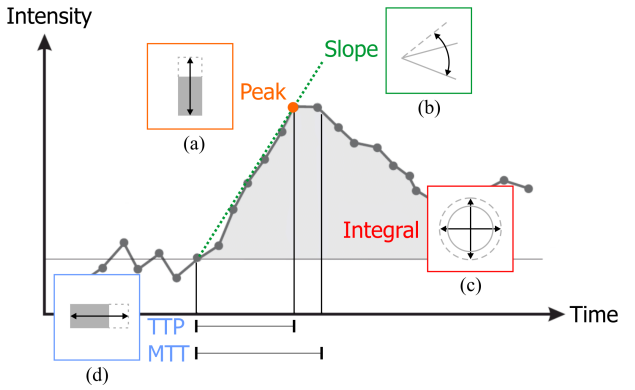

Fig. 1. A typical time-intensity curve in cerebral perfusion annotated with the essential parameters. Glyph attributes which are suitable for an intuitive mapping of parameters are indicated in (a)-(d), e.g., TTP should be mapped to the horizontal extension similar to a time axis, Slope to orientation and Integral to size.

Feature Specification. The diagnostic evaluation of the multi-field parameter data is challenging and time-consuming due to its complexity. Hence, we integrate a feature-based approach to direct the user to suspicious areas. Motivated by the work of Doleisch et al. [10], we apply the concept of linking \& brushing. A scatterplot opposing two perfusion parameters is linked to the glyph display. Once an interesting subset of the parameter data is interactively brushed in the plot, the glyph visualization is restricted to this subset. To account for the uncertainty involved in the definition of the subset, smooth brushing is supported. In contrast to a binary decision, this allows a gradual course from uninteresting (context) to definitely interesting (focus). This course is integrated in the glyph display by mapping the so-called degree of interest (DOI) to a glyph attribute.

Glyph Design and Placement. We decide in favor of a $2 \mathrm{D}$ slice-based glyph placement considering that medical scientists are used to a slice-based exploration. The $2 \mathrm{D}$ glyph shapes are presented within the context of an original 
slice. The slice is rendered as a texture on a $2 \mathrm{D}$ plane and scaled such that a single texel (texture element similar to a pixel in an image) covers more than one pixel on the screen. The glyphs are laid out texel-wise and slightly jittered about the texel centers to avoid visual irritations caused by grid patterns. To facilitate an intuitive mapping, we implemented several glyph shapes with different suitable visual attributes besides color (Fig. 1): circular discs (size), rectangles (horizontal and vertical extension), ellipses (size, orientation), toroids (size, roundness, thickness), and oriented toroids (equal to toroids plus orientation).

Multi-resolution Glyph Display. Initial tests showed that a texel-wise glyph placement results in very small glyphs due to the limited space per texel. For example, a screen area $S_{x y}$ of $512 \times 512$ pixels reserved for the texture display of a dataset matrix $M_{x y}=128 \times 128$ leads to an upper glyph size bound $B$ of $S_{x} / M_{x} \times S_{y} / M_{y}=4 \times 4$ pixels. This glyph size prevents the readability of subtle changes in glyph shape. A straightforward uniform scaling leads to overlapping artifacts. Hence, we implemented a multi-resolution glyph-display incorporating different resolution layers. The bottommost layer $L_{0}$ represents the highest resolution with one glyph per texel. For the above mentioned example, $B_{0}=4 \times 4$ pixels. In each following layer $L_{i}, i \in[1, n]$, a glyph covers the area of four quadratically arranged neighboring texels in $L_{i-1}$. A useful maximum number of layers $n$ has been determined empirically so far. For our example, $n$ has been set to three. With $B_{i}=2^{i} \cdot S_{x} / M_{x} \times 2^{i} \cdot S_{y} / M_{y}, i \in[1, n]$ pixels, this results in upper glyph size bounds $B_{1}=8 \times 8, B_{2}=16 \times 16$, and $B_{3}=32 \times 32$ pixels. For lower resolution layers, the parameter values which determine the glyph attributes have to be computed. Simple averaging of voxel values may suppress pathologies. Instead, we propose to compute the minimum or maximum value dependent on the particular parameter and the application. In tumor perfusion, e.g., high "Slope" values indicate suspicious tissue. Hence, the maximum value is coded in the next lower resolution layer. The user may interactively explore the layers starting with the initially displayed topmost layer.

\section{Results}

First, a cerebral perfusion dataset from ischemic stroke diagnostics has been analyzed (Fig.2, (a)-(c)). In (a), one circular disc has been placed per texel. The glyph placement is restricted to regions with high TTP and small Peak values (delayed and diminished perfusion). This restriction has been defined via smooth brushing to detect the infarcted tissue. While the glyph color is easily distinguishable, changes in size are hard to evaluate. Zooming in, as illustrated by the inlet, improves the readability but involves a loss of context information and spatial orientation. Therefore, we applied our multi-resolution glyph display (b). The layer with $B_{2}=16 \times 16$ pixels is presented, i.e. a glyph represents $4 \times 4$ voxels. Since the glyph size is modified according to Integral, whose small values are of special interest in cerebral perfusion, the minimum voxel value is mapped to size. Though the representation of small values by small glyphs is intuitive, interesting regions should immediately grab the viewers attention. Therefore, 
the mapping is inverted. In (c), the discs are replaced by rectangles since this primitive better fills in the rectangular region it represents. The glyph color is modified based on the respective DOI value.

Furthermore, a DCE mammography has been analyzed (Fig.2, (d)). Ellipses are used to code the two most relevant parameters in tumor perfusion. The

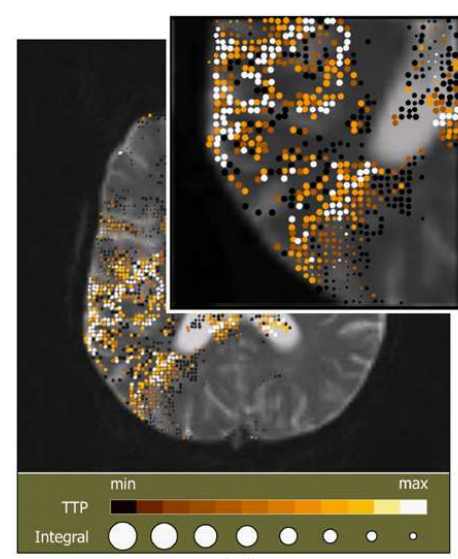

(a)

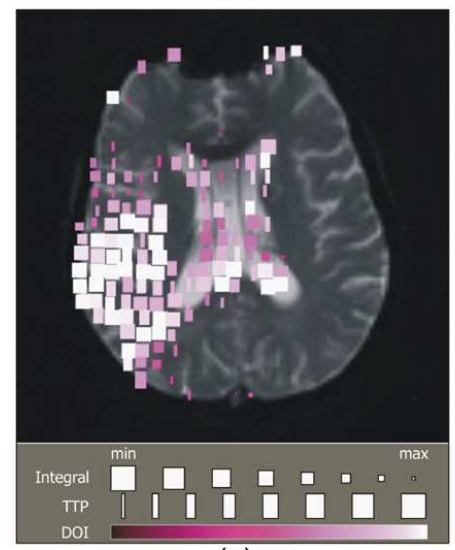

(c)

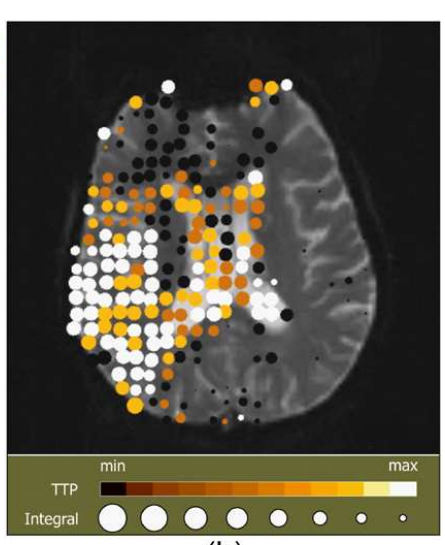

(b)

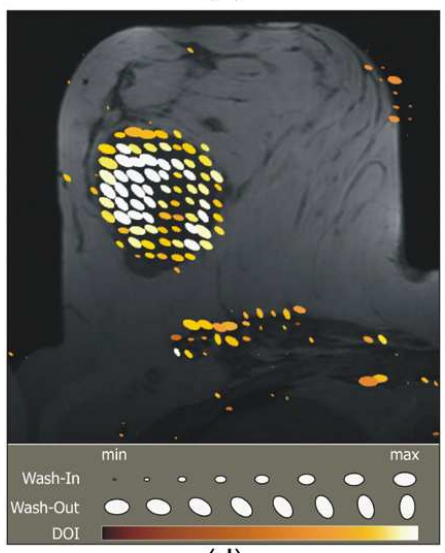

(d)

Fig. 2. Visualization examples based on the analysis of a cerebral perfusion $((a)-(c))$ and a DCE mammography dataset $(d)$. The glyph display in all images has been restricted to suspicious regions by means of smooth brushing. In $(a)$, one circular disc is placed per texel. Changes in glyph size are hard to interpret. A magnification (inlet) improves the readability but involves a loss of context information and spatial orientation. The application of a lower resolution layer $\left(B_{2}\right)$ solves the problem $(b)$. In $(c)$, the discs are replaced by rectangles since this primitive better fills in the rectangular region it represents. The DOI values are integrated by a color mapping. In $(d)$, ellipses are used to code the two most important parameters in tumor perfusion. Probably malignant tissue is indicated by large, vertically oriented ellipses. 
Slope (also called Wash-In in tumor diagnostics) is mapped to size while the velocity of the CA Wash-Out is mapped to orientation. The glyph placement is restricted to regions with large Wash-In values (suspicious enhancement). Probably malignant tissue is indicated by large, vertically oriented ellipses which represent strong perfusion and a high vessel permeability. In (d), the resolution layer with $B_{3}=32 \times 32$ pixels is presented, i.e. a glyph represents $8 \times 8$ voxels. The glyph size is modified according to the maximum voxel value.

\section{Discussion}

The developed glyph visualizations have been applied for detecting pathologies in three datasets so far. Initial tests indicated that, in spite of the multi-resolution display, some glyph attributes, such as roundness and thickness of toroids, are hard to interpret. Furthermore, the inverse mapping violates our concept of an intuitive mapping. To examine if our hypothesis of an easy to interpret coding of TIC shape is still supported, a clinical evaluation will be carried out.

Acknowledgement. We thank J.Wiener (Boca Raton Comm. Hosp., Florida) for providing the image data and $\mathrm{MeV}$ is Research for providing advanced MeVisLab features.

\section{References}

1. Choyke PL, Dwyer AJ, Knopp MV. Functional tumor imaging with dynamic contrast-enhanced magnetic resonance imaging. J Magn Reson Imaging. 2003 May;17(5):509-20.

2. König M, Klotz E, Heuser L. Perfusion CT in acute stroke: Characterization of cerebral ischemia using parameter images of cerebral blood flow and their therapeutic relevance. Electromedica. 1998;66(2):61-7.

3. Kohle S, Preim B, Wiener J, et al. Exploration of time-varying data for medical diagnosis. Proc VMV. 2002; p. 31-8.

4. Oeltze S, Grothues F, Hennemuth A, et al. Integrated visualization of morphologic and perfusion data for the analysis of coronary artery disease. Proc EuroVis. 2006; p. $131-8$.

5. Kindlmann G. Superquadric tensor glyphs. Proc VisSym. 2004; p. 147-54.

6. Wünsche BC, Lobb R, Young AA. The visualization of myocardial strain for the improved analysis of cardiac mechanics. Proc GRAPHITE. 2004; p. 90-9.

7. Paasche L, Oeltze S, Hennemuth A, et al. Integrierte Visualisierung kardialer MRDaten zur Beurteilung von Funktion, Perfusion und Vitalität des Myokards. Proc BVM. 2007; p. 212-6.

8. Ropinski T, Specht M, Meyer-Spradow J, et al. Surface glyphs for visualizing multimodal volume data. Proc VMV. 2007; p. to appear.

9. Rueckert D, Sonoda LI, Hayes C, et al. Nonrigid registration using free-form deformations: Application to breast MR images. IEEE Trans Med Imaging. 1999;18(8):712-21.

10. Doleisch H, Gasser M, Hauser H. Interactive feature specification for focus+context visualization of complex simulation data. Proc VisSym. 2003; p. 239-48. 